\title{
Examining perceptual and conceptual set biases in multiple-target visual search
}

\author{
Adam T. Biggs • Stephen H. Adamo • Emma Wu Dowd • \\ Stephen R. Mitroff
}

Published online: 14 February 2015

(C) The Psychonomic Society, Inc. 2015

\begin{abstract}
Visual search is a common practice conducted countless times every day, and one important aspect of visual search is that multiple targets can appear in a single search array. For example, an X-ray image of airport luggage could contain both a water bottle and a gun. Searchers are more likely to miss additional targets after locating a first target in multiple-target searches, which presents a potential problem: If airport security officers were to find a water bottle, would they then be more likely to miss a gun? One hypothetical cause of multiple-target search errors is that searchers become biased to detect additional targets that are similar to a found target, and therefore become less likely to find additional targets that are dissimilar to the first target. This particular hypothesis has received theoretical, but little empirical, support. In the present study, we tested the bounds of this idea by utilizing "big data" obtained from the mobile application Airport Scanner. Multiple-target search errors were substantially reduced when the two targets were identical, suggesting that the first-found target did indeed create biases during subsequent search. Further analyses delineated the nature of the biases, revealing both a perceptual set bias (i.e., a bias to find additional targets with features similar to those of the firstfound target) and a conceptual set bias (i.e., a bias to find additional targets with a conceptual relationship to the firstfound target). These biases are discussed in terms of the implications for visual-search theories and applications for professional visual searchers.
\end{abstract}

Keywords Visual search · Multiple-target search · Perceptual set . Conceptual set

A. T. Biggs $(\bowtie) \cdot S$. H. Adamo • E. W. Dowd $\cdot$ S. R. Mitroff Department of Psychology \& Neuroscience, Center for Cognitive Neuroscience, Duke University, B203 LSRC, Box 90999, Durham, NC 27708, USA

e-mail: adam.t.biggs@gmail.com
Visual search, or looking for targets amongst distractors, is a common activity conducted as part of everyday life. People conduct mundane visual searches, such as looking for soda in the refrigerator, as well as critically important searches, such as looking for explosives or guns in airport carry-on luggage. These searches - whether mundane or important — can be affected by a wide variety of top-down and bottom-up factors, including age differences (e.g., Humphrey \& Kramer, 1997; Madden, 1986), professional experience (e.g., Biggs, Cain, Clark, Darling, \& Mitroff, 2013; Biggs \& Mitroff, 2014), target-distractor similarity (e.g., Duncan \& Humphreys, 1989), and the type of search being conducted (e.g., Treisman \& Gelade, 1980). With such a wide variety of influences, visual search offers an ideal paradigm for investigating different aspects of visual and attentional abilities.

In most visual-search experiments, only one target is present during a trial (for recent reviews, see Eckstein, 2011; Nakayama \& Martini, 2011). However, real-world visualsearch scenarios can often have more than one target present in a single search. For example, more than one tumor can be present during a radiological screening, and more than one prohibited item can be present in a bag going through an airport security checkpoint. When visual search can include more than one target, a searcher typically must continue looking after finding a first target as it is often critical to find all targets present (e.g., finding one tumor but missing a second could jeopardize a patient's well-being). The potential presence of multiple targets introduces an important source of error in that a first-found target can cause searchers to miss additional targets (e.g., Berbaum, 2012). This phenomenon, an increased risk of missing additional targets after finding a first one, has previously been called the "satisfaction of search" effect (Smith, 1967; Tuddenham, 1962), but here, as we explain below, we refer to it as the "subsequent search misses" effect (SSM; Adamo, Cain, \& Mitroff, 2013). 
SSM errors have proven to be a persistent and stubborn source of error, accounting for as many as one-third of radiological misses under certain conditions (Anbari, 1997; Berbaum, Franklin, Caldwell, \& Schartz, 2010; Krupinski, 2010). These errors may be difficult to eliminate, in part, because their cause is multifaceted as opposed to arising from a single source (e.g., Berbaum et al., 2010; Cain, Adamo, \& Mitroff, 2013). For example, the original explanation-and the source of the original name - claimed that searchers became "satisfied" with the meaning of the search upon locating a first target and terminated their search prematurely (Smith, 1967; Tuddenham, 1962); however, more recent evidence has shown that searchers do continue searching after a first-found target (e.g., Berbaum et al., 1991; Berbaum et al., 1990; Fleck, Samei, \& Mitroff, 2010). Another proposed explanation for SSM errors is a resource depletion account (Berbaum et al., 1991; Cain \& Mitroff, 2013), which suggests that valuable cognitive resources (e.g., attention and working memory) are consumed by the found target. For example, a searcher might need to maintain the identity and location of the first-found target, thereby consuming attention and/or working memory resources, while leaving fewer resources available during subsequent search.

A third possible explanation for SSM errors is that searchers become biased to find additional targets similar to the first-found target (Berbaum et al., 1991; Berbaum et al., 1990; Berbaum et al., 2010). This account is the primary focus of the present study, and here we will refer to this hypothesized mechanism as the "similarity bias" account. ${ }^{1}$ The idea comes from academic radiology, and suggests that searchers become biased by the first-found target and primarily look for additional targets similar to the first when they resume searching - thus hindering the detection of features relevant to a different diagnosis. A similarity bias would likewise suggest that if an airport security officer located a water bottle in an X-ray image of a passenger's bag, he or she would then become biased to locate additional water bottles (or similar beverage containers) during subsequent search at the cost of detecting dissimilar targets, such as guns.

The similarity bias account shares some aspects in common with a priming influence on attention (e.g., Driver \& Tipper, 1989; Logan, 1980; Meyer \& Schvaneveldt, 1971). A priming-like account would suggest that finding a first target generates mental activation for the perceptual and semantic features of that target as search continues. As such, it is possible that a first-found target could create a "perceptual set" for similar physical features and/or a "conceptual set" for

\footnotetext{
${ }^{1}$ Note that this hypothesized explanation was originally referred to as the "perceptual set" account (e.g., Berbaum et al., 1991; Berbaum et al., 1990), but we have relabeled it here, given that we examine both a perceptual and a conceptual aspect to the effect (see below). We have opted to use a more generic label that can allow for SSM errors being affected by a perceptual bias, a conceptual bias, or both.
}

semantically related objects. Either possibility could then reduce the cost of searching for multiple target types (e.g., Godwin et al., 2010; Menneer, Cave, \& Donnelly, 2009; Menneer, Donnelly, Godwin, \& Cave, 2010; Stroud, Cave, Menneer, \& Donnelly, 2012). Critically, such similarity biases provide processing benefits for only certain classes of targets (i.e., those that share the key similarity) and do so to the detriment of other target types.

Some evidence has already suggested a strong role for perceptual similarity in guiding search. For example, more detailed visual information effectively guides search (Vickery, King, \& Jiang, 2005), and distractors are most distracting when they are visually similar to the target (Duncan \& Humphreys, 1989; Godwin, Hout, \& Menneer, 2014; Roper, Cosman, \& Vecera, 2013). The first-found target in a multipletarget search could potentially lead to perceptual priming of similar features due to the visual similarity between target items (cf. Wilschut, Theeuwes, \& Olivers, 2014). Conceptual similarity, on the other hand, has generally been found to have a small influence on visual-search performance (e.g., Godwin et al., 2014). For example, a recent study (Goodhew, Kendall, Ferber, \& Pratt, 2014) showed that activating a conceptual relation, such as semantic concepts (e.g., the color word "red"), led to increased attentional capture by visual cues that matched those concepts (e.g., a red-colored distractor). However, it is important to note that this conceptual relationship is very direct and limited to the same level of categorization. Furthermore, the links between these previous studies and multiple-target visual search are limited given that the previous tasks all involved single-target paradigms.

Several studies, in both academic radiology and cognitive psychology, have described the similarity bias as a potential cause of SSM errors in multiple-target searches, despite limited empirical data to support the idea (e.g., Ashman, Yu, \& Wolfman, 2000; Cain et al., 2013; Fleck et al., 2010). In one study (Fleck et al., 2010), participants looked for targets in the shape of a "T" amongst distractors in the shape of an "L." Any trial could have no, one, or two targets present and in one particular experiment (Fleck et al., 2010, Exp. 10), the target "T"s on dual-target trials were identical to one another (except for possibly their orientation). Their goal was to investigate whether SSM errors were affected by target similarity, in which two identical targets could potentially eliminate SSM errors during subsequent search after a found target. However, a significant SSM effect was found despite both targets being identical (Fleck et al., 2010). This example, and others, do not support a similarity bias account, although there has yet to be an investigation directly aimed at testing the validity and boundaries of a similarity bias in multiple-target search.

In line with the above example, additional data could be considered inconsistent with a similarity bias account. In radiology, significant SSM errors were observed even when multiple targets in the same search array shared perceptual 
features and belonged to the same general diagnosis (Ashman et al., 2000). However, it is critical to note that the continuing presence of SSM errors - even when two targets in the same search array have perceptual or conceptual similarities - is not sufficient to disprove the existence of a similarity bias. SSM errors have a wide variety of causes (e.g., Cain et al., 2013), and so another influence could be responsible for inducing significant SSM errors despite perceptual or conceptual similarity between targets. Thus, evidence in favor of a similarity bias account could simply show a reduction in SSM errorsnot necessarily a total elimination of them - when two targets share perceptual or conceptual features versus when two targets do not share these features.

Although previous investigations may have provided little or no evidence for a similarity bias account in multiple-target search, this lack of evidence might have been due to the small number of possible target items used in the experiments. For example, Fleck et al. (2010) had observers search for target "T"s amongst distractor "L"s, and the target "T"s were either relatively dark, as compared to the other items, or relatively light. If a similarity bias operates via a priming-like influence, such a design might be suboptimal, because the priming of the two target types could become chronic - repeated exposure to two target types could result in elevated activation across trials (i.e., you only need to see so many lightly colored T-shaped targets before you become generally biased toward lightly colored T-shaped targets across the entire experiment). As such, it could be difficult to properly measure the priminglike influence of one target type versus another if both have an equally elevated state of activation. A large and unpredictable set of targets could generate more short-lived priming during a given visual-search trial, which is more in line with the real-world scenarios in which a similarity bias could meaningfully impact performance.

In the present study, we utilized a large target set (79 different targets) with millions of experimental trials obtained through the mobile application Airport Scanner-a game wherein the player serves as an airport security officer and searches for "illegal" targets in simulated X-ray images of luggage (see Fig. 1 for examples). Two targets appeared simultaneously in approximately $6 \%$ of the simulated bags within the dataset obtained from the game, which allowed for investigations into SSM errors without biasing the participants to expect more than one target on any given trial. Furthermore, this varied target set and large number of data provided substantial power for examining potential similaritybased influences across a wide variety of first- and secondtarget combinations.

To examine the potential role of a similarity bias on SSM errors, we conducted three sets of analyses. First, we assessed the strongest possible influence of a similarity bias by comparing trials with two identical targets (e.g., a given bag with two identical pistols) to trials with two distinct targets (e.g., a

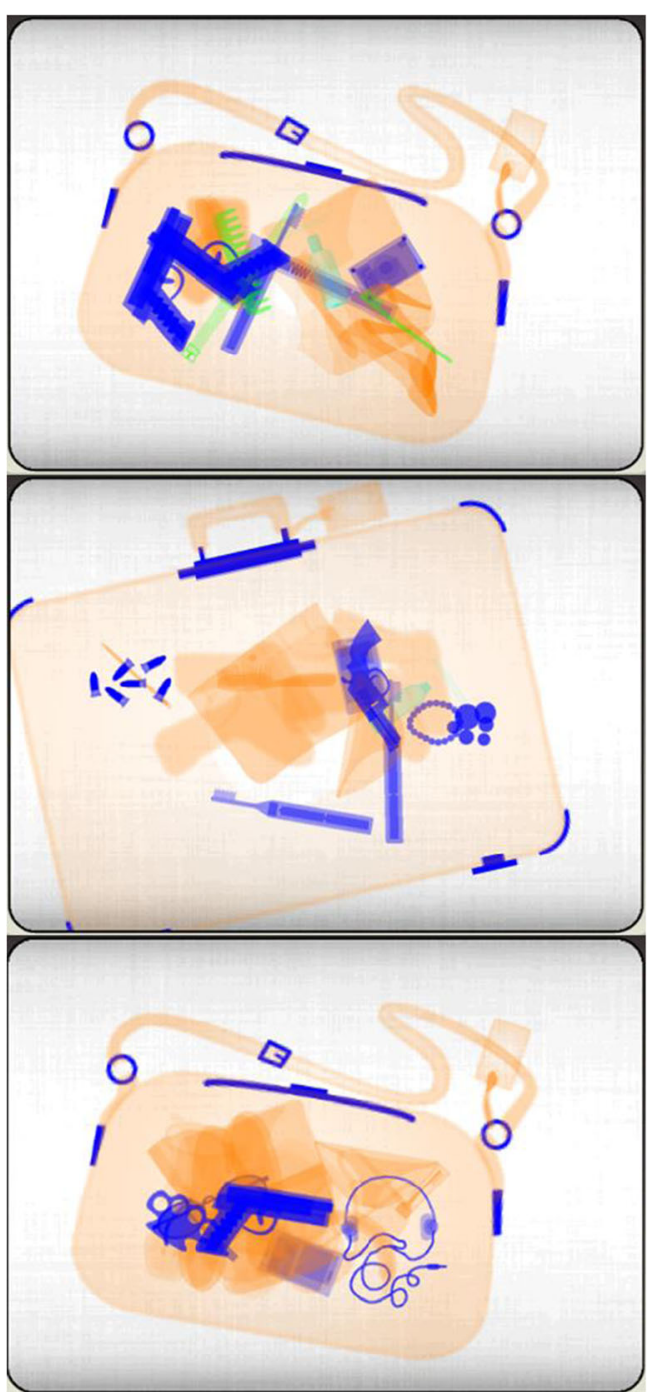

Fig. 1 Examples of multiple-target bags from Airport Scanner. The images include a bag with two identical targets (top, two pistols), a bag with two conceptually similar targets (middle, a revolver and bullets), and a bag with two targets that do not share a strong conceptual relationship (bottom, a pistol and brass knuckles)

given bag with one pistol and one oversized water bottle). If there is a bias to find a second target that is similar to the first (regardless of the nature of the similarity), then this comparison should reveal a significant difference in the rate of SSM errors when the targets were identical as compared to when they were not identical (see Mitroff et al., 2014). This test stands as the strongest possible assessment of a similarity bias because two identical targets share all perceptual and conceptual aspects in common (except perhaps physical orientation in the Airport Scanner implementation).

Second, we assessed a "perceptual set bias"- the propensity to look for additional targets that share physical features such as color, size, or shape in common with the found target. The Airport Scanner gameplay provided a simplified means to assess a possible perceptual set bias as each "illegal" target 
item was drawn in a single color. Thus, we were able to test a perceptual set bias by comparing SSM errors when two targets either were drawn in the same color or were drawn in different colors. Below we provide an assessment of a potential perceptual set bias with target color as the primary classifier and an additional assessment that reduces the potential influence of conceptual similarities between targets.

Finally, we assessed a "conceptual set bias"-the propensity to look for additional targets that had a similar purpose, function, or categorical relationship with the found target. Target items in Airport Scanner varied in conceptual as well as perceptual relationships, which provided an opportunity to compare the rate of SSM errors between cases when two targets were conceptually related and when two targets were not conceptually related. Notably, items with conceptual similarity often share physical attributes, as well (e.g., items in the category of "things you can sit on" are generally related to one another in terms of their size, shape, orientation, etc.). This was true in the present dataset, and so several steps were taken to minimize the influence of perceptual features when assessing a possible conceptual set bias, as we describe below in the Method section.

\section{Method}

A subset of the present analyses and results have been briefly presented elsewhere (Mitroff et al., 2014); however, the previous treatment was cursory and did not include full details of the methods, results, and interpretations. The previous use of these data was primarily done for illustrative purposes in an observation piece focused on the use of "big data" for research purposes.

\section{Overview}

Data collection was accomplished using the mobile application Airport Scanner for both Apple and Android platforms. Anonymous gameplay data were recorded in accordance with the terms and conditions of the standard Apple User Agreement and those provided by Kedlin Co. (https://www. airportscannergame.com). Each player consented to the terms and conditions voluntarily upon installing the Airport Scanner mobile application, and Kedlin Co. made the data available to our research team. The Duke University Institutional Review Board approved the analytical investigation of the dataset.

In the Airport Scanner game, players served as airport security officers who were primarily tasked with finding illegal items in simulated X-ray bags. Players viewed a single bag at a time and reported the presence of illegal items via finger taps directly onto each illegal item. At the time of data analysis for this study, the data pool consisted of 1.1 billion trials; however, far fewer trials contributed to the present analyses, since these data were filtered on the basis of the criteria described below. More information on the nature of these data can be found in other manuscripts based on the same mobile application (e.g., Biggs, Adamo, \& Mitroff, 2014; Mitroff \& Biggs, 2014; Mitroff et al., 2014).

\section{Participants}

Players advanced through five skill levels during gameplay, from Trainee to Elite, though only Elite players were included in the present analyses, to provide some level of assurance that each player had sufficient familiarity with the gameplay, because they would have had to complete a minimum of 618 trials to obtain Elite status (and most had significantly more exposure than that). Data from 62,606 unique devices contributed to the present analyses, and the data were collapsed across players, with no player-specific analyses being conducted.

\section{Airport scanner gameplay}

Players identified illegal target items during search by tapping a finger directly onto the item. Items appeared in one of several different bag types that varied in size, shape, and orientation, and each search display consisted of a single bag that moved from left to right on a conveyor belt. Each bag contained 0-3 "illegal" target items and 0-20 "legal" items that served as distractors. There were multiple levels (airports), which consisted of multiple sessions (days), and each session included multiple trials (bags).

Players could obtain in-game upgrades to make gameplay easier, with specific upgrades performing a variety of functions (e.g., speeding up or slowing down the conveyor belt). We eliminated the data collected when such upgrades were active, except for two classes of upgrades that did not impact the performance related to our present questions: Recharge Boosts, which helped recharge other upgrades for more frequent use, and the Rare Item Magnet, which attracted a special class of targets with in-game value, but this class of targets was not included in, or relevant to, any of the present analyses.

\section{Target and distractor stimuli}

The total pool of items included 94 possible target items and 94 possible distractor items that could be present during search. Our analyses focused on the target items that could appear in bags alongside legal distractors without requiring special in-game upgrades to view, which provided a pool of 79 possible target items. All items (both targets and distractors) were drawn in a semitransparent way, so that they could overlap without completely occluding one another. The size, color, and identity of the targets were all determined by Kedlin Co. for gameplay reasons and were designed to 
approximate the type of displays seen in airport security screening.

Both target and distractor items were introduced slowly into gameplay as players advanced through the various levels. For example, a player with minimal experience might only have an existing pool of five targets and 20 distractors. However, Elite players saw the full contingent of targets and distractors, which could appear at any point. The present dataset came from only Elite players, who had prior exposure to all the illegal and legal items.

\section{Accuracy trials}

Accuracy data were assessed for trials collected between April 15 and August 26, 2013. These data were filtered to focus our analyses, and the general data-filtering plan created a dataset that contained data only from Elite players, at any airport (game level) except the Trainee level, and included only bag types that could contain both illegal items that served as targets and legal items that served as distractors (i.e., bag types that could never contain a legal distractor item were not analyzed), bags that were not searched while utilizing in-game upgrades (except for those that did not affect visual search during gameplay; see above), and bags that did not contain "special" items such as the air marshal badge (whose presence indicated that players were to withhold responding to present targets) or unique target items with special in-game value (referred to as "Rare items" in the gameplay). The data filtering yielded two distinct datasets: one accuracy dataset with single-target trials, to use for baseline comparisons, and one accuracy dataset with dual-target trials, through which SSM errors could be assessed. Bags that contained zero or three targets were not analyzed as part of this dataset.

The single-target accuracy data included 1,795,907 trials (for more details on the single-target trials, see Biggs et al., 2014). Single-target accuracy was determined as the percentage of trials on which a particular target type was successfully detected out of all trials in which that target type had been the only illegal item present in the bag. Each single-target accuracy value was calculated from a sufficient number of cases, such that the values should be reliable and stable (range $=703$ to 114,390 appearances, $M=22,733$ appearances, $S E=3,724$ appearances).

\section{SSM calculations}

To measure SSM errors, we compared search accuracy for a specific target item on single-target trials to search accuracy for the same target on dual-target trials when the other target had been found first (e.g., Biggs \& Mitroff, 2014). Significant SSM errors occurred if there was a significant difference between single-target accuracy and accuracy on dual-target trials after a first target had already been found. Players identified a target by tapping directly onto it, yet a single finger tap could capture more than one target. For cases in which a single tap captured two targets (as determined by trials with two illegal items that had identical response times for detection), this could have been due to either the player's intent to identify two targets or the player's intent to identify one target and accidentally capturing the second target. To ensure that players intended to identify separate targets with each finger tap, we excluded all dual-target trials in which one tap identified more than one target. This data filtering produced 126, 579 valid dual-target trials for the analysis of SSM errors after all data trimming, including at least 136 appearances for every target item, with a maximum of 15,086 appearances $(M=3$, 205 appearances, $S E=440$ appearances).

The Airport Scanner gameplay varies target frequency, such that some targets were more likely to appear than others (see Mitroff \& Biggs, 2014). Approximately $50 \%$ of the bags contained at least one target, but the frequency of any given target appearing in a bag ranged in the present dataset from a high of $4.14 \%$ to a low of $0.078 \%$. These frequency differences, in turn, affected the likelihood of certain first- and second-target combinations appearing in dual-target trials (i.e., if two specific target types did not appear often in gameplay, there would not be many dual-target trials in which both items would appear together). We restricted the SSM error analyses to only target combinations with a sufficient number of occurrences, to best create a reliable accuracy dataset. We set the cutoff at 20 useable cases, on the basis of several previous rare-target search studies that had used 20 data points as a viable count (Fleck \& Mitroff, 2007; Russell \& Kunar, 2012; Wolfe, Horowitz, \& Kenner, 2005; Wolfe et al., 2007). Note that this data filtering was applied to all analyses presented here, which is why the degrees of freedom varied across analyses. For example, dual-target trials only rarely contained two identical targets (dual-target trials with identical targets $=4.34 \%$, dual-target trials with different targets $=95.66 \%$ ), which limited the dataset to 33 targets available for that particular analysis. In contrast, for assessing overall SSM errors, 78 of 79 targets had at least 20 trials that met all of the inclusion criteria $(M=1,602$ valid trials, $S E=$ 227 valid trials).

\section{Results}

As we noted in the Method section, a subset of the present results have been reported elsewhere (Mitroff et al., 2014). The overall SSM error rate and the identical- versus nonidentical-target SSM error rate data were briefly presented in Mitroff et al. for illustrative purposes. 


\section{Overall SSM errors}

SSM errors were the key focus of this investigation, and thus the first step of the analyses was to determine whether SSM errors occurred during Airport Scanner gameplay. The overall SSM error rate was calculated as the difference between the search accuracy for a given target on single-target trials and the search accuracy for that same item on dual-target trials, given that another target had been found first (e.g., Adamo et al., 2013; Biggs \& Mitroff, 2014; Cain \& Mitroff, 2013). This initial analysis was performed across 78 target items without regard to the identity of the first-found target on any given search trial; for example, when calculating the SSM error rate for the pistol as a second target, all data were included, regardless of whether the first-found target was another pistol, a large water bottle, a knife, and so forth. The overall SSM error rate was significant in a paired-samples $t$ test between the single-target accuracy for a given item and the dualtarget accuracy for that same item when another target had been found first $(M=14.00 \%, S E=1.11 \%), t(77)=12.62$, $p<.001$, indicating that Airport Scanner is a suitable paradigm for investigating SSM errors.

\section{Identical first and second targets}

The strongest possible test of whether a found target can bias subsequent search is a comparison between when the first and second targets (a) were and (b) were not identical. This comparison contrasts the case in which two identical targets shared every possible perceptual and conceptual connection with the case in which the two targets differed along at least one dimension. Note that two characteristics of the present dataset could introduce subtle differences between what we are calling "identical" items: Each item was presented in a random orientation in the picture plane, and each item could be occluded by distractor items. These two variables surely added some noise that would often make two identical targets not perfectly identical, but nevertheless, the targets were a perfect match in all other respects (size, shape, color, orientation in depth-plane, and conceptual attributes).

We observed significant SSM errors both when the first and second targets were identical $(M=6.53 \%, S E=1.62 \%)$, $t(32)=4.04, p<.001$, and when the first and second targets were not identical $(M=19.21 \%, S E=1.36 \%)$, $t(32)=14.13, p<.001$. However, the difference in the SSM error rates for identical versus nonidentical targets was also significant (mean difference $=12.69 \%, S E=1.69 \%$ ), $t(32)=7.52, p<.001 ; \mathrm{SSM}$ errors occurred when the two targets were identical, yet these errors were substantially reduced, relative to when the two targets were not identical.

One potential confound when comparing the SSM error rates for identical and nonidentical targets is that there were variable frequency rates across target items (the individual target frequencies ranged from a high of $4.14 \%$ to a low of $0.078 \%$ ). A bag was thus more likely to have two identical targets if the target in question was relatively common rather than relatively infrequent (i.e., if the pistol is more likely to appear than the ice pick, a bag is more likely to have two pistols than two ice picks). Consequently, the analysis of identical versus nonidentical targets disproportionately included more relatively common items in the identical-target data than in the nonidentical-target data. More frequent items were more likely to be detected (Biggs et al., 2014; Mitroff \& Biggs, 2014), and so the identical-target benefit (i.e., reduced SSM errors for two identical targets) could simply be an artifact of target frequency.

We examined this potential confound by comparing the size of the identical-target benefit for a particular target item (i.e., SSM errors with a different first target item minus SSM errors with an identical first target item) to the frequency of that target. A significant relationship emerged between the size of the identical-target benefit and the individual target frequency, $r(31)=-.43, p=.01$, such that infrequently appearing targets benefited more than did frequently appearing targets from an identical found target. The direction of this relationship, however, was opposite to what a frequency confound would predict - that the infrequently appearing targets would be less likely to be detected-suggesting that the identical-target benefit was not an artifact of target frequency.

This first set of analyses suggested that a first-found target can create biases during subsequent search that reduce- - but do not eliminate-SSM errors. The key question then became whether these biases are due to perceptual similarity, conceptual similarity, or both. The following analyses delved further into the nature of these biases to address this question. Note that all of the following analyses excluded trials with two identical targets.

\section{Perceptual set bias}

A perceptual set bias suggests that a found target biases searchers to find additional targets with similar perceptual features during subsequent search. Theoretically, any perceptual feature could bias search by means of this mechanism. Practically, here we focused on the target items' color as the principal perceptual feature. We did so because color is known to be a clear distinguishing feature in visual search that can effectively be used to discriminate targets from distractors (e.g., Biggs \& Gibson, 2010, 2013, 2014; Folk \& Remington, 1998; Gibson \& Bryant, 2008; Theeuwes \& Burger, 1998; Treisman \& Gelade, 1980; Turatto \& Galfano, 2000). Likewise, color serves as a prominent feature of targets in Airport Scanner and provides a straightforward means to perceptually categorize target items. All target items were drawn in a single color and in one of five colors: dark blue 
$(N=40)$, pink $(N=17)$, orange $(N=12)$, light blue $(N=8)$, and green $(N=2)$. Note that all of the following analyses excluded trials with two identical targets, which allowed for a direct examination of perceptual set biases without the influence of two identical targets.

For the present purposes, we focused analyses on the dark blue and pink items, since these two colors included the most target items. ${ }^{2}$ Our primary question of interest was whether SSM errors would be reduced significantly when two targets in the same bag were perceptually matched (i.e., the first and second targets were either both dark blue or both pink), as compared to when the two targets were not perceptually matched (i.e., one target was dark blue and the other target was pink). The shoe bomb target item (from the pink items) was not included in the analyses, because it had less than the 20 -trial minimum for reliable analyses. A $2 \times 2$ analysis of variance (ANOVA; see Table 1) on SSM errors with Color as a between-items factor (dark blue or pink second target) and Color Match as a within-items factor (first target same or different color) revealed a marginally nonsignificant main effect of color, with fewer SSM errors for dark blue targets $(M=$ $13.86 \%, S E=1.31 \%)$ than for pink targets $(M=18.27 \%, S E$ $=2.07 \%), F(1,54)=3.23, p=.08, \eta_{\mathrm{p}}{ }^{2}=.06$. We found a significant main effect of color match, with fewer SSM errors when the first and second targets were of the same color $(M=$ $14.70 \%, S E=1.22 \%)$ versus different colors $(M=17.44 \%$, $S E=1.33 \%), F(1,54)=16.37, p<.001, \eta_{\mathrm{p}}{ }^{2}=.23$. The interaction between these two variables was nonsignificant, $F(1,54)=2.08, p=.16$. The main effect of color match (and lack of a significant interaction or main effect of color) suggests that a perceptual set bias was created by the firstfound target during subsequent search; the color of the firstfound target primed searchers to find additional targets with the same color, versus additional targets with a different color.

Although the analysis above suggests a perceptual set influence on SSM errors that was driven by target color, it is important to note that color is not necessarily independent of target category. Color can systematically vary along with category membership for the current stimuli, so to fully evaluate a perceptual set account, it is necessary to minimize the

\footnotetext{
${ }^{2}$ The results and interpretations were similar when using only the dark blue and pink targets (as reported here) versus using targets of all five colors. We focused our perceptual set bias analyses to only the dark blue and pink targets because this offered the most straightforward test of the bias that was also compatible with the conceptual set bias reported below. For example, the eight light blue targets were all liquid containers, and thus could not be adequately divided between light blue items that either did or did not have conceptual similarity. When using targets of all five colors, we observed significant SSM errors when the first and second targets were the same primary color $(M=13.15 \%, S E=1.13 \%), t(72)$ $=7.67, p<.001$, and when the first and second targets were not the same primary color $(M=15.96 \%, S E=1.22 \%), t(72)=13.08, p<.001$; however, we also observed a significant difference in SSM error rates for two targets of the same color versus two targets of different colors (mean difference $=2.82 \%, S E=0.60 \%), t(72)=5.48, p<.001$.
}

Table 1 "Subsequent search miss" errors as a function of the color match between the first and second targets

\begin{tabular}{llll}
\hline & \multicolumn{2}{c}{ Color Match With First Target } & \\
\cline { 2 - 3 } $\begin{array}{l}\text { Second-Target } \\
\text { Color }\end{array}$ & Different Color & Same Color & Comparison \\
\hline (A) Perceptual Set Bias & & & \\
Dark blue & $14.74 \%(1.42 \%)$ & $12.98 \%(1.30 \%)$ & $1.76 \%$ \\
$\quad$ targets & $20.13 \%(2.24 \%)$ & $16.41 \%(2.06 \%)$ & $3.72 \%$ \\
$\begin{array}{l}\text { Pink targets } \\
\text { Blue \& pink } \\
\text { combined }\end{array}$ & $17.44 \%(1.33 \%)$ & $14.70 \%(1.22 \%)$ & $2.74 \%$ \\
(B) Perceptual Set Bias When Controlling for Conceptual Set & \\
$\begin{array}{c}\text { Dark blue } \\
\text { targets }\end{array}$ & $15.47 \%(1.80 \%)$ & $13.32 \%(1.79 \%)$ & $2.15 \%$ \\
$\begin{array}{c}\text { Pink targets } \\
\text { Blue \& pink } \\
\text { combined }\end{array}$ & $20.75 \%(2.92 \%)$ & $18.05 \%(2.91 \%)$ & $2.70 \%$ \\
\hline
\end{tabular}

Results are presented for (A) a test of a perceptual set bias while not controlling for conceptual similarities, and (B) a test of a perceptual set bias when controlling for conceptual similarities (i.e., two blue targets that were never both guns, or two pink targets that were never both explosives).

potential role of target category. In the present analysis, targets were divided into categories in addition to their separation by primary color (see Fig. 2). The target items were divided into two broad categories based on conceptual relationships-items related to guns and items related to explosives. This division by color and concept allowed for a perceptual set analysis limited to only targets that did not have a semantic relationship (i.e., trials with two dark blue targets were excluded if both targets were gun-related, and trials with two pink targets were excluded if both targets were explosives). It is also possible that items with clear conceptual identities could bias subsequent search toward that concept (i.e., a shotgun could prime a broad conceptual set for all guns), so to conduct the most conservative assessment of a perceptual set account, here we omitted trials if either a gun-related item or an explosive-related item was the first-found target. The gun part \#2, tommy gun, and virus-filled syringe target items (from the dark blue items) and cherry bombs, illegal pills, and shoe bomb target items (from the pink items) were not included in the analyses because they had less than the 20-trial minimum for reliable analyses.

A $2 \times 2$ ANOVA on SSM errors (see Table 1) with Color as a between-items factor (dark blue or pink second target) and Color Match as a within-items factor (first target same color or first target different color) revealed a nonsignificant main effect of color, with fewer SSM errors for dark blue targets than for pink targets, $F(1,49)=2.47, p=.12$. The main effect of color match was marginally significant, $F(1,49)=3.84$, $p=.06, \eta_{\mathrm{p}}{ }^{2}=.07$, with fewer SSM errors when the two target colors matched $(M=15.68 \%, S E=1.71 \%)$ than 


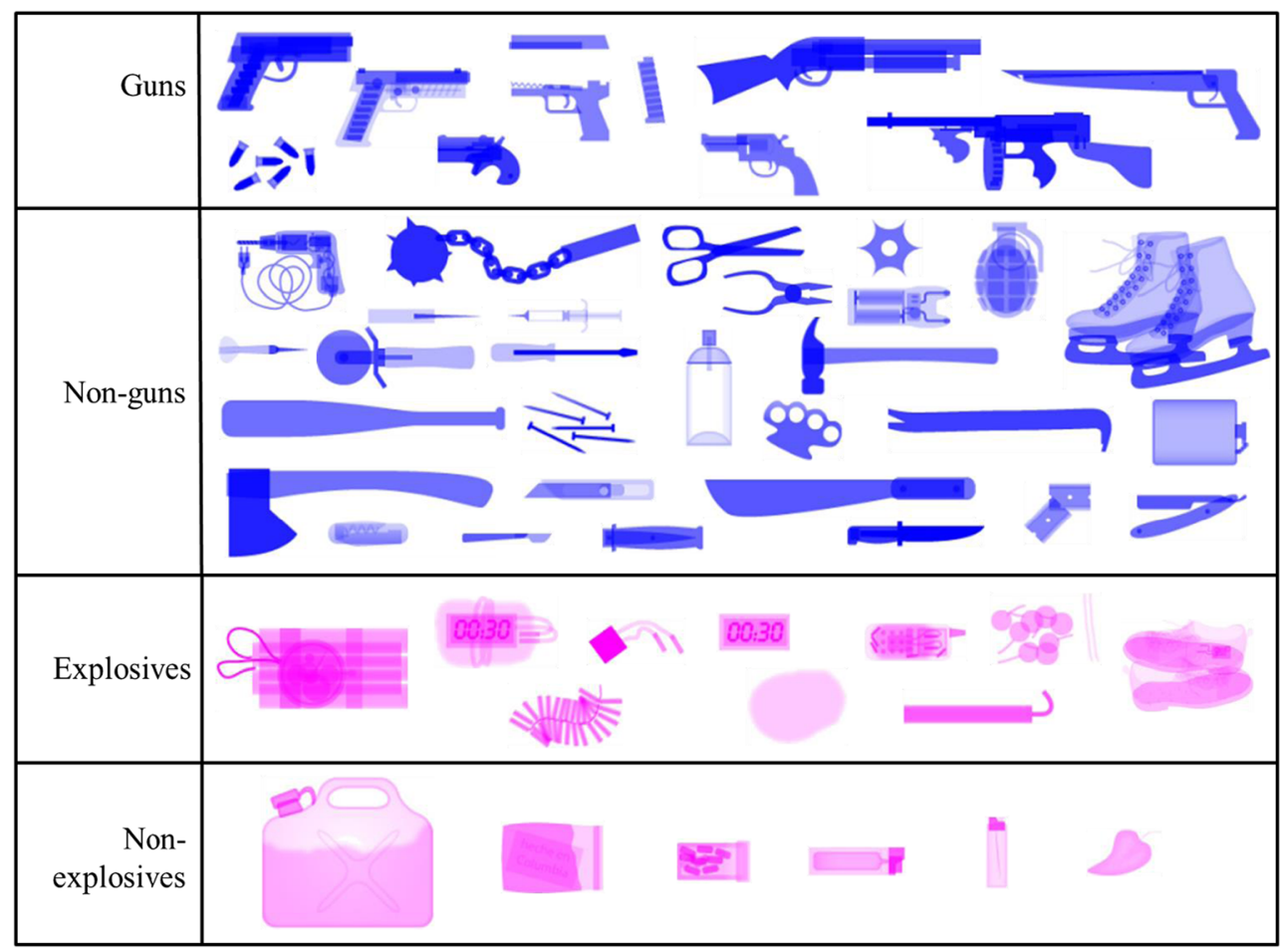

Fig. 2 Dark blue and pink target items in Airport Scanner gameplay, divided into distinct categories. Guns include (from left to right) the pistol, bullets, semi-polymer gun, derringer, gun part \#1, gun part \#2, gun part \#3, revolver, shotgun, tommy gun, and harpoon gun. Nonguns include (from left to right, starting at the top left) the electric drill, morning star, scissors, eight-inch pliers, ninja star, tazer, grenade, ice skates, dart, pizza cutter, ice pick, eight-inch screwdriver, virus-filled syringe, spray can, hammer, brass knuckles, crowbar, hip flask, aluminum bat, nails, axe, pocket knife, doctor's scalpel, box cutter,

when the two colors mismatched $(M=18.11 \%, S E=$ $1.72 \%)$. The interaction term was nonsignificant, $F(1,49)=$ $0.05, p=.83$. Thus, the perceptual set influence was reduced when controlling for conceptual influences. We will discuss this result more in the Discussion section.

Conceptual set bias A conceptual set bias suggests that a found target biases searchers to find additional targets with semantic, rather than perceptual, similarity to the first target. To test for a conceptual set bias and to control for overlap in perceptual features, we limited the analyses to only those instances in which the first and second targets shared the same color. Specifically, we focused solely on the gun-related items that were drawn in blue and the explosive-related items that were drawn in pink - that is, we compared (a) dark blue gunrelated items to dark blue non-gun-related items, and (b) pink explosive-related items to pink non-explosive-related items (see Fig. 2). Two particular targets were removed from these analyses: the shoe bomb and cherry bombs were pink explosives, and they were removed due to insufficient trials (i.e., less than the 20-trial minimum). The target set for these switchblade, machete, knife, razor blades, and straight razor. Explosives include (from left to right) the big bomb, small bomb, firecrackers, bomb part \#1, bomb part \#2, bomb part \#3, cell phone bomb, dynamite stick, cherry bombs, and shoe bomb. Note that the cherry bombs and shoe bomb targets were omitted from the conceptual set analyses due to too few contributing trials. Nonexplosives include (from left to right) the gasoline can, drugs, illegal pills, mace, lighter, and killer hot pepper

analyses yielded 11 dark blue gun-related items and 10 pink explosive-related items.

A $2 \times 2$ ANOVA (see Table 2) on SSM errors with Category as a between-items factor (gun vs. explosive) and Category Match as a within-items factor (first target same category and same color vs. first target different category and same color) revealed a marginally nonsignificant main effect of category, with fewer SSM errors in the gun category

Table 2 "Subsequent search miss" errors as a function of the category match between the first and second targets

\begin{tabular}{llll}
\hline & \multicolumn{2}{l}{ Category Match With First Target } & \\
\cline { 2 - 3 } $\begin{array}{l}\text { Second-Target } \\
\text { Category }\end{array}$ & $\begin{array}{l}\text { Different } \\
\text { Category }\end{array}$ & Same Category & Comparison \\
\hline $\begin{array}{l}\text { Gun-related items } \\
\begin{array}{l}\text { Explosive-related } \\
\text { items }\end{array}\end{array}$ & $13.65 \%(3.55 \%)$ & $9.60 \%(2.61 \%)$ & $4.05 \%$ \\
$\begin{array}{c}\text { Guns \& } \\
\text { explosives }\end{array}$ & $18.91 \%(2.58 \%)$ & $12.30 \%(1.89 \%)$ & $6.61 \%$ \\
\hline
\end{tabular}


$(M=11.71 \%, S E=2.78 \%)$ than in the explosive category $(M$ $=19.64 \%, S E=3.26 \%), F(1,17)=3.43, p=.08, \eta_{\mathrm{p}}{ }^{2}=.17$. There was a significant main effect of category match, with fewer SSM errors when the first and second targets were from the same category $(M=12.58 \%, S E=1.95 \%)$ than when they were from different categories $(M=18.77 \%, S E=2.65 \%)$, $F(1,17)=11.56, p<.01, \eta_{\mathrm{p}}{ }^{2}=.41$. The interaction between these factors was not significant, $F(1,17)=2.87, p=.11$.

This analysis suggests that the first-found target during visual search created a conceptual bias distinct from the above perceptual (color) bias. However, although we limited the influence of shared perceptual features in our conceptual set analyses by examining only first-and-second target combinations in which both targets shared the same color, the conceptually related items could also share other perceptual features besides color (e.g., shape). To further examine a conceptual set bias, we performed a follow-up analysis with specifically chosen stimuli that would help alleviate concerns about perceptual factors contributing to a conceptual set analysis. We focused on the bullets target stimulus, since it had a clear conceptual relationship with the other gun-related items, yet shared only the perceptual feature of color (as opposed to size or shape) with the other gun-related items. That is, in this stimulus set, the bullets had a conceptual relationship - but not size or shape similarity — with the other gun-related items. Taking advantage of this situation, we assessed the potential for a conceptual bias in SSM errors by examining accuracy at detecting bullets as a second target when either (a) any dark blue gun-related item was the first target, as compared to when (b) any dark blue non-gun-related item was the first target. We conducted a $2 \times 2$ chi-square analysis (see Table 3 ) for bullets as a second target with accuracy (hit vs. miss) as the first dimension and found target (any dark blue gun vs. any dark blue nongun) as the second dimension. This analysis revealed a significant increase in accuracy for bullets when the first target was a gun versus when the first target was not a gun, $\chi^{2}(1, N=4,762)=25.81, p<.001$, and suggests that, even when controlling for the only shared perceptual feature (color), a conceptual relationship between targets reduced SSM errors.

Table 3 Counts for bullets as a second target, based on the first-found target and detection of the bullets

\begin{tabular}{lll}
\hline & \multicolumn{2}{l}{ First-Found Target } \\
\cline { 2 - 3 } Bullets As Second Target & Any Blue Gun & Any Blue Nongun \\
\hline Bullets target detected & $876(62.71 \%)$ & $1,839(54.65 \%)$ \\
Bullets target missed & $521(37.29 \%)$ & $1,526(45.35 \%)$ \\
\hline
\end{tabular}

Rows represent the numbers of cases in which bullets were either detected or missed when they occurred as a second target (with the relative percentage of cases, based on the first target, in parentheses)

\section{Discussion}

Visual searches are routinely conducted in a wide variety of contexts - some of which are mundane, and others of which are critically important. One interesting aspect of visual search, particularly for searches conducted in real-world settings, is that more than one target can be present within a single search. This multiple-target aspect compels the searcher to continue looking for additional targets after finding a first target, which provides the opportunity to explore several cognitive influences of the first-found target. Here, we examined the biases created by the first-found target during multipletarget search through the use of the mobile application Airport Scanner - a simulated airport screening task embedded within a videogame platform that contains a varied target set and provided us with millions of trials.

Previous work has suggested a potential similarity-based influence in multiple-target search, wherein a searcher becomes biased to look for additional targets similar to the first-found target (e.g., Berbaum et al., 2010). This bias was hypothesized to lead to an increase in SSM errors, such that searchers become less likely to find subsequent targets that do not match the found target. Although this is a viable hypothesis that has been offered since at least 1990 (Berbaum et al., 1991; Berbaum et al., 1990), minimal empirical data have been offered in support of it (e.g., Ashman et al., 2000; Cain et al., 2013; Fleck et al., 2010; see Mitroff et al., 2014, for a discussion of this point).

The goal of the present study was to use a large dataset to look for evidence of a similarity bias in multiple-target visual search. Such a bias can take one of several forms, and we subdivided this broader concept into two distinct components: a perceptual set bias, which directs attention toward other items that share physical features with a found target, and a conceptual set bias, which directs attention toward other items that share a conceptual relationship with a found target.

Our findings first established that a found target created biases during subsequent search, by demonstrating significantly reduced SSM errors when the first and second targets were identical, as compared to when they were different items (see also Mitroff et al., 2014). Next, we found evidence for both perceptual and conceptual set biases, such that SSM errors were reduced when the second target shared either perceptual or conceptual features with the first-found target. Further analyses revealed that the conceptual set bias contributed as a significant influence on SSM errors when controlling for possible perceptual influences, but that the perceptual set bias produced a smaller influence when controlling for possible conceptual influences (see Fig. 3). Previous evidence from single-target visual-search tasks had suggested that a perceptual set bias is a likely contributor to visual-search accuracy (e.g., Godwin et al., 2014; Wilschut et al., 2014)—and therefore potentially to SSM errors - so the marginal effect here 


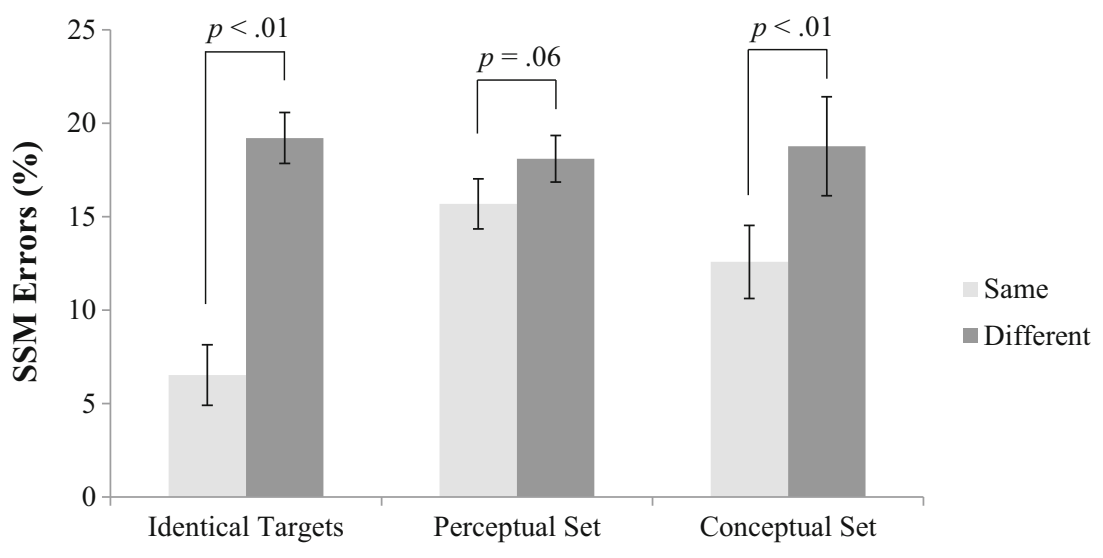

Subsequent Search Bias

Fig. 3 Subsequent search miss (SSM) errors, separated for the three primary biases investigated here and whether the first-/second-target combination matched according to each bias ("same") or mismatched ("different"). Identical targets represents the analysis in which the first and second targets were the exact same item or different items. Perceptual set represents the analysis in which the first and second targets shared the same color versus had different colors, while limiting the possible

could be due to limiting the perceptual analyses to a single feature rather than multiple features (e.g., color alone rather than color and shape). That is, detailed target information is beneficial to visual search (Vickery, King, \& Jiang, 2005), and a perceptual set might benefit from incorporating multiple factors rather than a single factor.

As we mentioned earlier, a potential underlying mechanism of the similarity bias is priming, either perceptual or conceptual. Perceptual priming effects, in which repeated stimuli are identified better, are typically explained by a sharpening of the stimulus representation upon repeated exposure (see Wiggs \& Martin, 1998). Conceptual priming effects are based on the idea of spreading activation, in which the initial processing of a stimulus automatically spreads to semantically related stimuli (e.g., Collins \& Loftus, 1975). In both cases, finding a first target may reduce the processing threshold for other, perceptually or conceptually related stimuli, thus improving the detection of a similar second target (e.g., Kristjánsson \& Campana, 2010).

Another possible explanation for the similarity bias involves aspects of memory-guided attention (see Hutchinson $\&$ Turk-Browne, 2012). Several studies have shown that encoding and maintaining an item in working memory appears to bias attention toward memory-matching items in a search array (see Soto, Hodsoll, Rotshtein, \& Humphreys, 2008, and Olivers, Peters, Houtkamp, \& Roelfsema, 2011, for reviews; but see also Woodman \& Luck, 2007), above and beyond the influence of priming (Dowd \& Mitroff, 2013; Downing, 2000; Olivers, Meijer, \& Theeuwes, 2006; Soto, Heinke, Humphreys, \& Blanco, 2005). In the present study, participants actively processed and interacted with the first-found target, by explicitly tapping on the item. This influence of a semantic relationship between the two targets by removing conceptual relationships (see the main text and Table 1). Conceptual set represents the analysis in which the first and second targets were of the same color (diminishing perceptual relationships), but either did or did not have a semantic relationship. Thus, both the perceptual set and conceptual set analyses controlled for the orthogonal influence of either conceptual or perceptual similarity, respectively

conscious and explicit interaction with the first target (as opposed to the mere exposure that is typified by pure priming) may have encoded its perceptual and conceptual features into working memory (see Cain \& Mitroff, 2013). Maintaining these features in working memory could have then biased attention toward other items in the same array that shared those perceptual and/or conceptual features.

This hypothetical working memory account of similarity bias can be described in terms of the biased-competition model of attention (Desimone \& Duncan, 1995): A first-found target is encoded and actively maintained in working memory, resulting in top-down biasing of its perceptual and/or conceptual features. Due to the capacity limitations of attention, the biased-competition model also predicts the suppression of nonmatching perceptual and/or conceptual features. Attention then becomes preferentially allocated toward items with matching features and away from items with competing features, such that attentional resources are most efficiently distributed to task-relevant information. Although the present analyses do not address the idea of suppressing targets that have competing features, a biased-competition mechanism fits well with the resource depletion account of SSM errors (Berbaum et al., 1991; Cain \& Mitroff, 2013). Specifically, perceptual and conceptual set biases could arise as a result of a diminished pool of available resources: After finding a first target, searchers may best utilize their remaining cognitive resources by biasing attention in subsequent search toward the properties of the found target.

A similarity bias account of SSM errors also represents a logical extension of real-world practices conducted by professional visual searchers. For example, a radiological scan might depict chest trauma that resulted in several broken ribs. Any 
action that could inflict such trauma is also likely to have other predictable influences on the body, and radiologists may therefore strategically look for additional trauma consistent with the original injury - thus evoking a perceptual set bias, conceptual set bias, or both. Likewise, if an airport security officer finds a detonator for an explosive device, then it is logical to search for additional components that would be needed to make a functional bomb. It could, in fact, be highly beneficial to adapt a perceptual or conceptual set bias if one target indicates an above-average likelihood that another specific target might be present - though the benefit comes with a known trade-off. The Airport Scanner mobile application did not impose such necessary relationships (i.e., bullets could randomly appear with any other target, without a systematic link to a conceptually related item such as a gun); however, if such relationships are probable in a search environment, then the perceptual and conceptual set biases might become even more prominent than we had observed here. These higherlevel strategic influences likely contribute to SSM errors in practical searches, above and beyond the possible contribution of memory-based guidance.

In conclusion, a first-found target in a multiple-target visual search can create significant biases during subsequent search. This hypothesized cause of SSM errors had lived as a viable theoretical stance, but it had yet to be empirically demonstrated. Adding this fact to the existing literature further supports the suggestion that SSM errors are multifaceted in nature, rather than due to a single source (Berbaum et al., 2010; Cain et al., 2013). Furthermore, the present study has provided solid evidence for a conceptual influence in multiple-target visual search.

Author note We thank Ben Sharpe, Thomas Liljetoft, and Kedlin Company for access to the Airport Scanner data and for approving the use of Airport Scanner images in the manuscript. The dataset for the present project and a subset of the analyses and methods were previously reported in Mitroff et al. (2014). These previous analyses were based on a simplified version designed for illustrative purposes. This work was partially supported by Army Research Office Grant Nos. W911NF-13-10480 and W911NF-14-1-0361.

\section{References}

Adamo, S. H., Cain, M. S., \& Mitroff, S. R. (2013). Self-induced attentional blink: A cause of errors in multiple-target visual search. Psychological Science, 24, 2569-2574.

Anbari, M. M. (1997). Cervical spine trauma radiography: Sources of false-negative diagnoses. Emergency Radiology, 4, 218-224.

Ashman, C. J., Yu, J. S., \& Wolfman, D. (2000). Satisfaction of search in osteoradiology. American Journal of Roentgenology, 175, 541-544.

Berbaum, K. S. (2012). Satisfaction of search experiments in advanced imaging. Proceedings of SPIE, 8291(1), 82910V-82910V-8.

Berbaum, K. S., Franken, E. A., Jr., Dorfman, D. D., Rooholamini, S. A., Coffman, C. E., Cornell, S. H., \& Smith, T. P. (1991). Time course of satisfaction of search. Investigative Radiology, 26, 640-648.
Berbaum, K. S., Franken, E. A., Jr., Dorfman, D. D., Rooholamini, S. A., Kathol, M. H., Barloon, T. J., \& Montgomery, W. J. (1990). Satisfaction of search in diagnostic radiology. Investigative Radiology, 25, 133-140.

Berbaum, K. S., Franklin, E. A., Jr., Caldwell, R. T., \& Schartz, K. M. (2010). Satisfaction of search in traditional radiographic imaging. In E. Samei \& E. Krupinski (Eds.), The handbook of medical image perception and techniques (pp. 107-138). New York: Cambridge University Press.

Biggs, A. T., Adamo, S. H., \& Mitroff, S. R. (2014). Rare, but obviously there: Effects of target frequency and salience on visual search accuracy. Acta Psychologica, 152, 158-165.

Biggs, A. T., Cain, M. S., Clark, K., Darling, E. F., \& Mitroff, S. R. (2013). Assessing visual search performance differences between Transportation Security Administration Officers and nonprofessional searchers. Visual Cognition, 21, 330-352.

Biggs, A. T., \& Gibson, B. S. (2010). Competition between color salience and perceptual load during visual selection can be biased by topdown set. Attention, Perception, \& Psychophysics, 72, 53-64. doi: 10.3758/APP.72.1.53

Biggs, A. T., \& Gibson, B. S. (2013). Learning to ignore salient color distractors during serial search: Evidence for experience-dependent attention allocation strategies. Frontiers in Cognition, 4, 326. doi:10. 3389/fpsyg.2013.00326

Biggs, A. T., \& Gibson, B. S. (2014). Visual salience can co-exist with dilution during visual selection. Journal of Experimental Psychology: Human Perception and Performance, 40, 7-14. doi: 10.1037/a0033922

Biggs, A. T., \& Mitroff, S. R. (2014). Different predictors of multipletarget search accuracy between non-professional and professional visual searchers. Quarterly Journal of Experimental Psychology, 67, 1335-1348.

Cain, M. S., Adamo, S. H., \& Mitroff, S. R. (2013). A taxonomy of errors in multiple-target visual search. Visual Cognition, 21, 899-921.

Cain, M. S., \& Mitroff, S. R. (2013). Memory for found targets interferes with subsequent performance in multiple-target visual search. Journal of Experimental Psychology: Human Perception and Performance, 39, 1398-1408. doi:10.1037/a0030726

Collins, A. M., \& Loftus, E. F. (1975). A spreading-activation theory of semantic processing. Psychological Review, 82, 407-428. doi:10. 1037/0033-295X.82.6.407

Desimone, R., \& Duncan, J. (1995). Neural mechanisms of selective visual attention. Annual Review of Neuroscience, 18, 193-222. doi:10.1146/annurev.ne.18.030195.001205

Dowd, E. W., \& Mitroff, S. R. (2013). Attentional guidance by working memory overrides salience cues in visual search. Journal of Experimental Psychology: Human Perception and Performance, 39, 1786-1796.

Downing, P. E. (2000). Interactions between visual working memory and selective attention. Psychological Science, 11, 467-473. doi:10. 1111/1467-9280.00290

Driver, J., \& Tipper, S. P. (1989). On the nonselectivity of "selective" seeing: Contrasts between interference and priming in selective attention. Journal of Experimental Psychology: Human Perception and Performance, 15, 304-314. doi:10.1037/0096-1523.15.2.304

Duncan, J., \& Humphreys, G. W. (1989). Visual search and stimulus similarity. Psychological Review, 96, 433-458. doi:10.1037/0033295X.96.3.433

Eckstein, M. P. (2011). Visual search: a retrospective. Journal of Vision, 11(5), 14:1-36. doi:10.1167/11.5.14

Fleck, M. S., \& Mitroff, S. R. (2007). Rare targets are rarely missed in correctable search. Psychological Science, 18, 943-947.

Fleck, M. S., Samei, E., \& Mitroff, S. R. (2010). Generalized "satisfaction of search": Adverse influences on dual target search accuracy. Journal of Experimental Psychology: Applied, 16, 60-71. 
Folk, C. L., \& Remington, R. (1998). Selectivity in distraction by irrelevant featural singletons: Evidence for two forms of attentional capture. Journal of Experimental Psychology: Human Perception and Performance, 24, 847-858. doi:10.1037/0096-1523.24.3.847

Gibson, B. S., \& Bryant, T. A. (2008). The identity intrusion effect: Attentional capture or perceptual load? Visual Cognition, 16, 182199. doi:10.1080/13506280701467987

Godwin, H. J., Hout, M. C., \& Menneer, T. (2014). Visual similarity is stronger than semantic similarity in guiding visual search for numbers. Psychonomic Bulletin \& Review, 21, 689-695.

Godwin, H. J., Menneer, T., Cave, K. R., Helman, S., Way, R. L., \& Donnelly, N. (2010). The impact of relative prevalence on dualtarget search for threat items from airport X-ray screening. Acta Psychologica, 134, 79-84.

Goodhew, S. C., Kendall, W., Ferber, S., \& Pratt, J. (2014). Setting semantics: Conceptual set can determine the physical properties that capture attention. Attention, Perception, \& Psychophysics, 76, 1577-1589. doi:10.3758/s13414-014-0686-3

Humphrey, D. G., \& Kramer, A. F. (1997). Age differences in visual search for feature, conjunction, and triple-conjunction targets. Psychology and Aging, 12, 704-717.

Hutchinson, J. B., \& Turk-Browne, N. B. (2012). Memory-guided attention: Control from multiple memory systems. Trends in Cognitive Sciences, 16, 576-579.

Kristjánsson, Á., \& Campana, G. (2010). Where perception meets memory: A review of repetition priming in visual search tasks. Attention, Perception, \& Psychophysics, 72, 5-18. doi:10.3758/APP.72.1.5

Krupinski, E. A. (2010). Current perspectives in medical image perception. Attention, Perception, \& Psychophysics, 72, 1205-1217.

Logan, G. D. (1980). Attention and automaticity in Stroop and priming tasks: Theory and data. Cognitive Psychology, 12, 523-553. doi:10. 1016/0010-0285(80)90019-5

Madden, D. J. (1986). Adult age differences in the attentional capacity demands of visual search. Cognitive Development, 1, 335-363.

Menneer, T., Cave, K. R., \& Donnelly, N. (2009). The cost of search for multiple targets: Effects of practice and target similarity. Journal of Experimental Psychology: Applied, 15, 125-135.

Menneer, T., Donnelly, N., Godwin, H. J., \& Cave, K. R. (2010). High or low target prevalence increases the dual-target cost in visual search. Journal of Experimental Psychology: Applied, 16, 133-144.

Meyer, D. E., \& Schvaneveldt, R. W. (1971). Facilitation in recognizing pairs of words: Evidence of a dependence between retrieval operations. Journal of Experimental Psychology, 90, 227-234. doi:10. 1037/h0031564

Mitroff, S. R., \& Biggs, A. T. (2014). The ultra-rare-item effect: Visual search for exceedingly rare items is highly susceptible to error. Psychological Science, 25, 284-289.

Mitroff, S. R., Biggs, A. T., Adamo, S. H., Dowd, E. W., Winkle, J., \& Clark, K. (2014). What can 1 billion trials tell us about visual search? Journal of Experimental Psychology: Human Perception and Performance. doi:10.1037/xhp0000012

Nakayama, K., \& Martini, P. (2011). Situating visual search. Vision Research, 51, 1526-1537.

Olivers, C. N. L., Meijer, F., \& Theeuwes, J. (2006). Feature-based memory-driven attentional capture: Visual working memory content affects visual attention. Journal of Experimental Psychology: Human
Perception and Performance, 32, 1243-1265. doi:10.1037/00961523.32.5.1243

Olivers, C. N. L., Peters, J., Houtkamp, R., \& Roelfsema, P. R. (2011). Different states in visual working memory: When it guides attention and when it does not. Trends in Cognitive Sciences, 15, 327-334. doi:10.1016/j.tics.2011.05.004

Roper, Z. J., Cosman, J. D., \& Vecera, S. P. (2013). Perceptual load corresponds with factors known to influence visual search. Journal of Experimental Psychology: Human Perception and Performance, 39, 1340-1351.

Russell, N. C., \& Kunar, M. A. (2012). Colour and spatial cueing in lowprevalence visual search. Quarterly Journal of Experimental Psychology, 65, 1327-1344.

Smith, M. J. (1967). Error and variation in diagnostic radiology. Springfield: C. C. Thomas.

Soto, D., Heinke, D., Humphreys, G. W., \& Blanco, M. J. (2005). Early, involuntary top-down guidance of attention from working memory. Journal of Experimental Psychology: Human Perception and Performance, 31, 248-261. doi:10.1037/0096-1523.31.2.248

Soto, D., Hodsoll, J., Rotshtein, P., \& Humphreys, G. W. (2008). Automatic guidance of attention from working memory. Trends in Cognitive Sciences, 12, 342-348. doi:10.1016/j.tics.2008.05.007

Stroud, M. J., Cave, K. R., Menneer, T., \& Donnelly, N. (2012). Why is it difficult to search for two colors at once? How eye movements can reveal the nature of representations during multi-target search. Journal of Experimental Psychology: Human Perception and Performance, 38, 113-122.

Theeuwes, J., \& Burger, R. (1998). Attentional control during visual search: The effect of irrelevant singletons. Journal of Experimental Psychology: Human Perception and Performance, 24, 1342-1353. doi:10.1037/0096-1523.24.5.1342

Treisman, A. M., \& Gelade, G. (1980). A feature-integration theory of attention. Cognitive Psychology, 12, 97-136. doi:10.1016/00100285(80)90005-5

Tuddenham, W. J. (1962). Visual search, image organization, and reader error in roentgen diagnosis. Radiology, 78, 694-704.

Turatto, M., \& Galfano, G. (2000). Color, form and luminance capture attention in visual search. Vision Research, 40, 1639-1643.

Vickery, T. J., King, L.-W., \& Jiang, Y. (2005). Setting up the target template in visual search. Journal of Vision, 5(1), 8:81-92. doi:10. $1167 / 5.1 .8$

Wiggs, C. L., \& Martin, A. (1998). Properties and mechanisms of perceptual priming. Current Opinion in Neurobiology, 8, 227-233.

Wilschut, A., Theeuwes, J., \& Olivers, C. N. L. (2014). Priming and the guidance by visual and categorical templates in visual search. Frontiers in Psychology, 5, 148. doi:10.3389/fpsyg.2014.00148

Wolfe, J. M., Horowitz, T. S., \& Kenner, N. M. (2005). Rare items often missed in visual searches. Nature, 435, 439-440. doi:10.1038/ 435439a

Wolfe, J. M., Horowitz, T. S., Van Wert, M. J., Kenner, N. M., Place, S. S., \& Kibbi, N. (2007). Low target prevalence is a stubborn source of errors in visual search tasks. Journal of Experimental Psychology: General, 136, 623-638. doi:10.1037/0096-3445.136.4.623

Woodman, G. F., \& Luck, S. J. (2007). Do the contents of visual working memory automatically influence attentional selection during visual search? Journal of Experimental Psychology: Human Perception and Performance, 33, 363-377. doi:10.1037/0096-1523.33.2.363 